\title{
A Unified Presentation of Certain Classical Polynomials
}

\author{
By H. M. Srivastava and J. P. Singhal
}

\begin{abstract}
This paper attempts to present a unified treatment of the classical orthogonal polynomials, viz. Jacobi, Laguerre and Hermite polynomials, and their generalizations introduced from time to time. The results obtained here include a number of linear, bilinear and bilateral generating functions and operational formulas for the polynomials $\left\{T_{n}{ }^{(\alpha, \beta)}(x, a, b, c, d, p, r) \mid n=0,1,2, \cdots\right\}$, defined by Eq. (3) below.*
\end{abstract}

1. Introduction and Definition. In an attempt to give a unified presentation of the classical orthogonal polynomials, viz. Jacobi, Laguerre and Hermite polynomials, Fujiwara [4] studied the polynomials defined by the generalized Rodrigues' formula

$$
p_{n}(x)=\frac{(-c)^{n}}{n !}(x-a)^{-\alpha}(b-x)^{-\beta} D^{n}\left\{(x-a)^{n+\alpha}(b-x)^{n+\beta}\right\},
$$

where $D=d / d x$.

The polynomials $p_{n}(x)$ are orthogonal with respect to the weight function $(x-a)^{\alpha}(b-x)^{\beta}$, where $\alpha, \beta>-1$, over the interval $[a, b]$. In fact, as pointed out by Szegö himself [10, p. 58], they can be rewritten as (cf. also [2])

$$
p_{n}(x)=c^{n}(a-b)^{n} P_{n}^{(\alpha, \beta)}\left(2\left\{\frac{x-a}{a-b}\right\}+1\right),
$$

where $P_{n}^{(\alpha, \beta)}(x)$ is the classical Jacobi polynomial, orthogonal with respect to the weight function $(1-x)^{\alpha}(1+x)^{\beta}$, where $\alpha, \beta>-1$, over the interval $[-1,1]$. By recourse to certain limiting processes, it is easy to verify that the $p_{n}(x)$, as also the Jacobi polynomials $P_{n}^{(\alpha, \beta)}(x)$, give rise to Hermite and Laguerre polynomials.

It may be of interest to study here the polynomial system $\left\{T_{n}^{(\alpha, \beta)}(x, a, b, c, d, p, r)\right\}$ defined by

$$
T_{n}^{(\alpha, \beta)}(x, a, b, c, d, p, r)
$$

$$
=\frac{(a x+b)^{-\alpha}(c x+d)^{-\beta}}{n !} \exp \left(p x^{r}\right) D^{n}\left\{(a x+b)^{n+\alpha}(c x+d)^{n+\beta} \exp \left(-p x^{r}\right)\right\} .
$$

Received March 6, 1972.

AMS 1970 subject classifications. Primary 33A65, 33A45; Secondary 42A52.

Key words and phrases. Linear, bilinear and bilateral generating functions, orthogonal polynomials, Jacobi polynomials, Laguerre polynomials, Hermite polynomials, operational formulas, Bessel polynomials, Lagrange's theorem, Gegenbauer polynomials, Leibniz' rule, Taylor's theorem.

* See also Abstract 72T-B26, Notices Amer. Math. Soc., v. 19, 1972, p. A-302. This work was supported in part by the National Research Council of Canada under Grant A-7353.

Copyright @ 1972, American Mathematical Society 
Apart from being more general than $p_{n}(x)$, the polynomial system defined by (3) has a distinct advantage in that it can be specialized to almost all classes of orthogonal polynomials without using any limiting processes. In particular, we mention the following obvious connections.

$$
\begin{aligned}
T_{n}^{(\alpha, \beta)}(x, a,-a, c, c, 0, r) & =T_{n}^{(\alpha, \beta)}(x, a,-a, c, c, p, 0)=(2 c a)^{n} P_{n}^{(\alpha, \beta)}(x) \\
T_{n}^{(\alpha, \beta)}(x, a, 0,0, d, 1,1) & =(a d)^{n} L_{n}^{(\alpha)}(x) . \\
T_{n}^{(\alpha, \beta)}(x, 0, b, c, 0,1,1) & =(b c)^{n} L_{n}^{(\beta)}(x) . \\
T_{n}^{(\alpha, \beta)}(x, 0, b, 0, d, 1,2) & =(-b d)^{n} H_{n}(x) . \\
T_{n}^{(\alpha, 0)}(x, a, 0, c, 0,2,-1) & =(2 a c)^{n} Y_{n}^{(\alpha)}(x) . \\
T_{n}^{(\alpha-n, \beta)}(x, a, 0,0, d, p, r) & =\frac{(-a d x)^{n}}{n !} H_{n}^{r}(x, \alpha, p) .
\end{aligned}
$$

Here, $Y_{n}^{(\alpha)}(x)$ denotes the generalized Bessel polynomial of Krall and Frink [6] defined by

$$
Y_{n}^{(\alpha)}(x)={ }_{2} F_{0}\left[-n, n+\alpha+1 ;-;-\frac{1}{2} x\right],
$$

and $H_{n}^{r}(x, \alpha, p)$ is the generalized Hermite polynomial

$$
H_{n}^{r}(x, \alpha, p)=(-1)^{n} x^{-\alpha} \exp \left(p x^{r}\right) D^{n}\left\{x^{\alpha} \exp \left(-p x^{r}\right)\right\},
$$

introduced earlier by Gould and Hopper [5]. form

By making use of Leibniz' rule, $T_{n}^{(\alpha, \beta)}(x, a, b, c, d, p, r)$ can be expressed in the

$$
T_{n}^{(\alpha, \beta)}(x, a, b, c, d, p, r)=(a d-b c)^{n}
$$

$$
\cdot \sum_{k=0}^{n} \frac{(-)^{k}}{k !}\left\{\frac{(a x+b)(c x+d)}{a d-b c}\right\}^{k} P_{n-k}^{(\alpha+k, \beta+k)}\left(2 a \frac{c x+d}{a d-b c}-1\right) H_{n}^{r}(x, 0, p) .
$$

Moreover, it is readily seen that

$$
T_{n}^{(\alpha, \beta)}(x, a, b, c, d, p, r)=(a b)^{-n} T_{n}^{(\beta, \alpha)}\left(\frac{b c x}{a d}, a, b, a^{2} d, b^{2} c,\left(\frac{a d}{b c}\right)^{r} p, r\right),
$$

which is a generalization of the familiar result $[10$, p. 59]

$$
P_{n}^{(\alpha, \beta)}(-x)=(-)^{n} P_{n}^{(\beta, \alpha)}(x)
$$

involving Jacobi polynomials.

2. Generating Functions. From the definition (3), we have

$$
\begin{aligned}
\sum_{n=0}^{\infty}\left\{\frac{t}{(a x+b)(c x+d)}\right\}_{n}^{n} T_{n}^{(\alpha-\lambda n, \beta-\mu n)}(x, a, b, c, d, p, r) \\
=(a x+b)^{-\alpha}(c x+d)^{-\beta} \exp \left(p x^{r}\right) \\
\cdot \sum_{n=0}^{\infty} \frac{t^{n}}{n !} D^{n}\left\{(a x+b)^{n+\alpha-\lambda n}(c x+d)^{n+\beta-\mu n} \exp \left(-p x^{r}\right)\right\}
\end{aligned}
$$


and, on interpreting this last expression by means of Lagrange's theorem [7]

$$
\frac{f(y)}{1-t \phi^{\prime}(y)}=\sum_{n=0}^{\infty} \frac{t^{n}}{n !} D^{n}\left\{[\phi(x)]^{n} f(x)\right\}, \quad y=x+t \phi(y),
$$

we find that

$$
\begin{aligned}
\sum_{n=0}^{\infty} T_{n}^{(\alpha-\lambda n, \beta-\mu n)}(x, a, b, c, d, p, r) t^{n} & \left(\frac{a y+b}{a x+b}\right)^{\alpha}\left(\frac{c y+d}{c x+d}\right)^{\beta} \exp \left\{p x^{r}-p y^{r}\right\} \\
= & \frac{\left(\frac{a x+b}{a y+b}\right)^{\lambda}\left(\frac{c x+d}{c y+d}\right)^{\mu-1}-c t(1-\mu)(a x+b)\left(\frac{a x+b}{a y+b}\right)^{\lambda-1}\left(\frac{c x+d}{c y+d}\right)^{\mu}}{1-a t(1-\lambda)(c x+d)}
\end{aligned}
$$

where

$$
y=x+t(a x+b)(c x+d)\left(\frac{a x+b}{a y+b}\right)^{\lambda-1}\left(\frac{c x+d}{c y+d}\right)^{\mu-1} .
$$

If we put

$$
(a x+b) /(a y+b)=1+u, \quad(c x+d) /(c y+d)=1+v,
$$

then we shall get the generating function

$$
\begin{aligned}
\sum_{n=0}^{\infty} T_{n}^{(\alpha-\lambda n, \beta-\mu n)}(x, & a, b, c, d, p, r) t^{n} \\
& =\frac{(1+u)^{-\alpha}(1+v)^{-\beta}}{1+(1-\lambda) u+(1-\mu) v} \exp \left\{p x^{r}-p\left(\frac{a x-b u}{a+a u}\right)^{r}\right\},
\end{aligned}
$$

where $u$ and $v$ are given by

$$
\left\{\begin{array}{l}
u=-a t(c x+d)(1+u)^{\lambda}(1+v)^{\mu-1}, \\
v=-c t(a x+b)(1+u)^{\lambda-1}(1+v)^{\mu} .
\end{array}\right.
$$

Alternatively, by letting $u=-\xi /(1+\xi)$ and $v=-\eta /(1+\eta)$, the generating function (17) can be written as

$$
\begin{aligned}
\sum_{n=0}^{\infty} T_{n}^{(\alpha-\lambda n, \beta-\mu n)}(x, a, b, c, d, p, r) t^{n} & \\
& =\frac{(1+\xi)^{\alpha+1}(1+\eta)^{\beta+1}}{1+\lambda \xi+\mu \eta-(1-\lambda-\mu) \xi \eta} \exp \left\{p x^{r}-p\left(x+\frac{\xi}{a}(a x+b)\right)^{r}\right\},
\end{aligned}
$$

where

$$
\left\{\begin{array}{l}
\xi=a t(c x+d)(1+\xi)^{1-\lambda}(1+\eta)^{1-\mu}, \\
\eta=c t(a x+b)(1+\xi)^{1-\lambda}(1+\eta)^{1-\mu}
\end{array}\right.
$$

In view of the relationships (4) through (9), the results given above would readily yield a large number of generating functions for the polynomials of Jacobi, Hermite, Laguerre, Gegenbauer, and many others. For instance, we have the following special cases: 
(i) If $\lambda=\mu=0$, then both (17) and (19) would give us the elegant generating function

$$
\begin{aligned}
\sum_{n=0}^{\infty} T_{n}^{(\alpha, \beta)}(x, & a, b, c, d, p, r) t^{n} \\
= & 2^{\alpha+\beta} \rho^{-1}\{1+(b c-a d) t+\rho\}^{-\alpha}\{1-(b c-a d) t+\rho\}^{-\beta} \\
& \cdot \exp \left\{p x^{r}-\frac{p}{a^{r}}\left[\frac{2(a x+b)}{1+(b c-a d) t+\rho}-b\right]^{r}\right\},
\end{aligned}
$$

where $\rho=\left\{1-2 t(b c+2 a c x+a d)+t^{2}(b c-a d)^{2}\right\}^{1 / 2}$.

(ii) For $p$ or $r=0$ and $-a / b=c / d=1,(17)$ and (19) correspond to our main results in [9], while (21) would give us the familiar generating function ([10, p. 69]; see also [7, Vol. 1, pp. 127, 303, Problem 219])

$$
\sum_{n=0}^{\infty} P_{n}^{(\alpha, \beta)}(x) t^{n}=2^{\alpha+\beta} R^{-1}(1-t+R)^{-\alpha}(1+t+R)^{-\beta},
$$

in which $R=\left(1-2 x t+t^{2}\right)^{1 / 2}$.

(iii) On combining (8) with (17) and (18), we get the known generating function [1, p. 654]

$$
\sum_{n=0}^{\infty} Y_{n}^{(\alpha+\lambda n)}(x) \frac{t^{n}}{n !}=(1+u)^{-\alpha}\{1+(2+\lambda) u\}^{-1} \exp \left\{t(1+u)^{-\lambda-1}\right\},
$$

where $-\frac{1}{2} x t=u(1+u)^{\lambda+1}$.

(iv) On using (9) with (19) and (20), we are led to the generating function

$$
\sum_{n=0}^{\infty} H_{n}^{r}(x, \alpha-\lambda n, p) \frac{t^{n}}{n !}=(1+\xi)^{\alpha+1}(1+\lambda \xi+\xi)^{-1} \exp \left[p x^{r}\left\{1-(1+\xi)^{r}\right\}\right],
$$

where $\xi=-x^{-1} t(1+\xi)^{-\lambda}$.

The last result (24) does not seem to have been noticed earlier.

3. Operational Formulas. In this section, we shall make use of the differential operator $\delta=x d / d x$ which possesses the following interesting properties:

$$
\begin{gathered}
x^{n} D^{n}=\delta(\delta-1) \cdots(\delta-n+1), \\
f(\delta) \exp \{g(x)\} h(x)=\exp \{g(x)\} f\left\{\delta+x g^{\prime}\right\} h(x) .
\end{gathered}
$$

Assuming $Y$ to be a sufficiently differentiable function and using the properties (25) and (26), we observe that

$$
\begin{aligned}
& (a x+b)^{-\alpha}(c x+d)^{-\beta} \exp \left(p x^{r}\right) D^{n}\left\{(a x+b)^{n+\alpha}(c x+d)^{n+\beta} \exp \left(-p x^{r}\right) Y\right\} \\
& =\left\{\frac{(a x+b)(c x+d)}{x}\right\}^{n} \prod_{j=1}^{n}\left[\delta+\frac{(n+\alpha) a x}{a x+b}+\frac{(n+\beta) c x}{c x+d}-p r x^{r}-j+1\right] Y,
\end{aligned}
$$

whereas, by employing Leibniz' rule, the left-hand side of (27) can also be expressed in the form

$$
n ! \sum_{k=0}^{n} \frac{(a x+b)^{k}(c x+d)^{k}}{k !} T_{n-k}^{(\alpha+k, \beta+k)}(x, a, b, c, d, p, r) D^{k} Y .
$$

Equivalence of the two expressions yields the operational formula 


$$
\begin{array}{r}
\prod_{j=1}^{n}\left[\delta+\frac{a(n+\alpha) x}{a x+b}+\frac{c(n+\beta) x}{c x+d}-p r x^{r}-j+1\right] Y \\
=n !\left\{\frac{x}{(a x+b)(c x+d)}\right\}^{n} \sum_{k=0}^{n} \frac{(a x+b)^{k}(c x+d)^{k}}{k !} \\
\cdot T_{n-k}^{(\alpha+k, \beta+k)}(x, a, b, c, d, p, r) D^{k} Y .
\end{array}
$$

For $Y=1$, (27) would give us

$$
\begin{aligned}
\prod_{j=1}^{n}\left[\delta+\frac{a(n+\alpha) x}{a x+b}+\right. & \left.\frac{c(n+\beta) x}{c x+d}-p r x^{r}-j+1\right] \cdot 1 \\
& =n !\left\{\frac{x}{(a x+b)(c x+d)}\right\}^{n} T_{n}^{(\alpha, \beta)}(x, a, b, c, d, p, r) .
\end{aligned}
$$

If in (29) we replace $n$ by $n+m$ and then interpret the left-hand side with the help of (28) and (29), we shall get the interesting formula

$$
\begin{aligned}
\left(\begin{array}{c}
m+n \\
n
\end{array}\right) T_{m+n}^{(\alpha, \beta)}(x, a, b, c, d, p, r)=\sum_{k=0}^{\infty} \frac{(a x+b)^{k}(c x+d)^{k}}{k !} \\
\cdot T_{n-k}^{(\alpha+k, \beta+k)}(x, a, b, c, d, p, r) D^{k} T_{m}^{(\alpha+n, \beta+n)}(x, a, b, c, d, p, r) .
\end{aligned}
$$

As an application of (30), we first replace $\alpha, \beta$ by $\alpha-n$ and $\beta-n$, respectively, multiply both sides by $t^{n}$, take their sums from $n=0$ to $n=\infty$ and then interpret its right-hand side with the help of Taylor's theorem and formula (18). We are thus led to the following generating function:

$$
\begin{aligned}
\sum_{n=0}^{\infty}\left(\begin{array}{c}
m+n \\
n
\end{array}\right) T_{m+n}^{(\alpha-n, \beta-n)}(x, a, b, c, d, p, r) t^{n} & \\
= & \{1+a t(c x+d)\}^{\alpha}\{1+c t(a x+b)\}^{\beta} \\
\cdot & \exp \left\{p x^{r}-p(x+t(a x+b)(c x+d))^{r}\right\} \\
& \cdot T_{m}^{(\alpha, \beta)}(x+t(a x+b)(c x+d), a, b, c, d, p, r) .
\end{aligned}
$$

On the other hand, by making use of the definition (3) and Lagrange's theorem (16), we observe that

$$
\begin{aligned}
& \sum_{n=0}^{\infty}\left(\begin{array}{c}
m+n \\
n
\end{array}\right) T_{m+n}^{(\alpha-n),}(x, a, b, c, d, p, r)\left(\frac{t}{a x+b}\right)^{n} \\
& =\frac{(a x+b)^{-\alpha}(c x+d)^{-\beta}}{(1-c t)^{m+1}} \exp \left(p x^{r}\right) \frac{1}{m !}\left(\frac{d}{d y}\right)^{m}\left\{(a y+b)^{\alpha+m}(c y+d)^{\beta+m} \exp \left(-p y^{r}\right)\right\},
\end{aligned}
$$

where $y=(x+d t)(1-c t)^{-1}$.

Interpreting the right-hand side of the last equation by means of (3), we get another generating function in the form

$$
\begin{aligned}
\sum_{n=0}^{\infty}\left(\begin{array}{c}
m+n \\
n
\end{array}\right) T_{m+n}^{(\alpha-n, \beta)}(x, a, b, c, d, p, r) t^{n} \\
\begin{aligned}
(32)= & \{1+t(a d-b c)\}^{\alpha}\{1-c t(a x+b)\}^{-\alpha-\beta-m-1} \\
& \cdot \exp \left\{p x^{r}-p\left(\frac{x+d t(a x+b)}{1-c t(a x+b)}\right)^{r}\right\} T_{m}^{(\alpha, \beta)}\left(\frac{x+d t(a x+b)}{1-c t(a x+b)}, a, b, c, d, p, r\right) .
\end{aligned}
\end{aligned}
$$


It is worthwhile to remark here that in view of relations (5) and (6), the generating function (32) provides us with a unification of a large number of results including, for instance, the known formulas (cf., e.g., [2, pp. 221-222])

$$
\sum_{n=0}^{\infty}\left(\begin{array}{c}
m+n \\
n
\end{array}\right) L_{m+n}^{(\alpha)}(x) t^{n}=(1-t)^{-\alpha-m-1} \exp \left(-\frac{x t}{1-t}\right) L_{m}^{(\alpha)}\left(\frac{x}{1-t}\right)
$$

and

$$
\sum_{n=0}^{\infty}\left(\begin{array}{c}
m+n \\
n
\end{array}\right) L_{m+n}^{(\alpha-n)}(x) t^{n}=(1+t)^{\alpha} \exp (-x t) L_{m}^{(\alpha)}\{x(1+t)\}
$$

4. Bilateral Generating Functions. In this section, we apply the generating relations (31) and (32) to prove the following theorems.

THEOREM 1. If

$$
F[x, t]=\sum_{n=0}^{\infty} a_{n} T_{n}^{(\alpha-n, \beta)}(x, a, b, c, d, p, r) t^{n},
$$

where the $a_{n}$ are arbitrary constants, then

$$
\{1+t(a d-b c)\}^{\alpha}\{1-c t(a x+b)\}^{-\alpha-\beta-1} \exp \left\{p x^{r}-p\left(\frac{x+d t(a x+b)}{1-c t(a x+b)}\right)^{r}\right\}
$$

$$
\cdot F\left[\frac{x+d t(a x+b)}{1-c t(a x+b)}, \frac{y t}{1+t(a d-b c)}\right]=\sum_{n=0}^{\infty} T_{n}^{(\alpha-n, \beta)}(x, a, b, c, d, p, r) \sigma_{n}(y) t^{n},
$$

where $\sigma_{n}(y)$ is a polynomial of degree $n$ in $y$ given by

$$
\sigma_{n}(y)=\sum_{k=0}^{n}\left(\begin{array}{l}
n \\
k
\end{array}\right) a_{k} y^{k}
$$

THEOREM 2. For arbitrary $a_{n}, n \geqq 0$, let.

$$
G[x, t]=\sum_{n=0}^{\infty} a_{n} T_{n}^{(\alpha-n, \beta-n)}(x, a, b, c, d, p, r) t^{n},
$$

then

$$
\begin{aligned}
&\{1+a t(c x+d)\}^{\alpha}\{1+c t(a x+b)\}^{\beta} \exp \left\{p x^{r}-p(x+t(a x+b)(c x+d))^{r}\right\} \\
& \cdot G[x+t(a x+b)(c x+d), y t /\{1+a t(c x+d)\}\{1+c t(a x+b)\}] \\
&= \sum_{n=0}^{\infty} T_{n}^{(\alpha-n, \beta-n)}(x, a, b, c, d, p, r) \sigma_{n}(y) t^{n},
\end{aligned}
$$

where $\sigma_{n}(y)$ is given by (37).

To prove Theorem 1 , we substitute the series expansion of $\sigma_{n}(y)$, given by (37), on the right-hand side of (36) and we get

$$
\begin{aligned}
\sum_{n=0}^{\infty} T_{n}^{(\alpha-n, \beta)}(x, & a, b, c, d, p, r) \sigma_{n}(y) t^{n} \\
& =\sum_{k=0}^{\infty} a_{k} y^{k} t^{k} \sum_{n=0}^{\infty}\left(\begin{array}{c}
n+k \\
n
\end{array}\right) T_{n+k}^{(\alpha-n-k, \beta)}(x, a, b, c, d, p, r) t^{n}
\end{aligned}
$$


On summing the inner series with the help of (32) and then interpreting the resulting expression by means of (35), we are led immediately to Theorem 1 .

Similar is the proof of Theorem 2. Indeed, we make use of the generating relation (31) in place of (32).

Alternatively, Theorems 1 and 2 may be deduced as corollaries of a general result on bilateral generating functions given elsewhere by us [8].

Department of Mathematics

University of Victoria

Victoria, B. C., Canada

Department of Mathematics

University of Jodhpur

Jodhpur, India

1. Léon-Claude Calvez \& Robert Génin, "Sur les rélations entre les fonctions génératrices et les formules de type Rodrigues," C. R. Acad. Sci. Paris Sér. A-B, v. 269, 1969, pp. A651-A654. MR 41 \#2080.

2. L. CARLITZ, "A note on the Laguerre polynomials," Michigan Math. J., v. 7, 1960, pp. 219-223. MR 22 \#11159.

3. S. K. ChatterJea, "On the unified presentation of classical orthogonal polynomials," SIAM Rev., v. 12, 1970, pp. 124-126. MR 41 \#5670.

4. I. FuJIWARA, "A unified presentation of classical orthogonal polynomials," Math. Japon., v. 11, 1966, pp. 133-148. MR 35 \#3106.

5. H. W. Gould \& A. T. HOPPER, "Operational formulas connected with two generalizations of Hermite polynomials, Duke Math. J., v. 29, 1962, pp. 51-63. MR 24 \# A2689.

6. H. L. KRALL \& O. FrinK, "A new class of orthogonal polynomials: The Bessel polynomials," Trans. Amer. Math. Soc., v. 65, 1949, pp. 100-115. MR 10, 453

7. G. Pólya \& G. Szegö, Aufgaben und Lehrsätze aus der Analysis, Springer, Berlin, 1925 ; reprint, Dover, New York, 1945.

8. H. M. SRIVAStaVa \& J. P. Singhal, "A class of bilateral generating functions for certain classical polynomials," Pacific J. Math., v. 42, 1972; Notices Amer. Math. Soc., v. 18, 1971, p. 515. Abstract \#684-B14.

9. H. M. SRivastava \& J. P. Singhal, "New generating functions for Jacobi and related polynomials," J. Math. Anal. Appl. (To appear.); Notices Amer. Math. Soc., v. 18, 1971, p. 555. Abstract \#71T-B83.

10. G. Szegö, Orthogonal Polynomials, Amer. Math. Soc. Colloq. Publ., vol. 23, Amer. Math. Soc., Providence, R. I., 1939. MR 1, 14. 